\title{
Traumatic handlebar hernia: case report of rare type of abdominal wall hernia
}

\author{
Mohamedsuror Baderalmaarif ${ }^{1}$, Faisal A. Nugud ${ }^{1}$ and Awad Ali M. Alawad ${ }^{2 *}$ \\ ${ }^{1}$ Department of Surgery, Faculty of Medicine, University of Gezira, Sudan \\ ${ }^{2}$ Department of Surgery, Faculty of Medicine, University of Medical Sciences and Technology, Sudan
}

\begin{abstract}
Acute Handlebar hernia is a rare type of hernia which might be a source of anxiety to the family as well as it could be associated with serious abdominal injuries. Here we reported a case of this type. He is a boy of 3 years presented to emergency department after sustaining a blunt abdominal trauma by a handlebar. The final diagnosis was confirmed using U/S and surgical treatment was achieved.
\end{abstract}

\section{Introduction}

Traumatic abdominal wall hernia is a rare clinical entity despite the high incidence of blunt abdominal trauma. The first reported case was in 1906. Few cases were reported in the literature [1]. Handlebar hernias, which are localized defects, are even more infrequent. Only 42 cases reported from September 1977 to November 2010 [2]. It involves disruption of the abdominal wall muscles, with bowel loop herniated through the defect in the abdominal wall, and may have major or even lethal complications [2]. We report a case of handlebar hernia a male child of 3 years, who came for treatment 2 days after the injury. Ultrasonography confirmed the clinical diagnosis of traumatic hernia. The case was taken up for open surgery and anatomical repair was done with prolene. The patient had an uneventful postoperative course.

\section{Case report}

A 3 -year old male was brought to our department with a reducible swelling measuring $6 \times 4 \mathrm{~cm}$ in the left side of lower abdomen after sustaining a trauma by a bicycle handlebar, as it fell on his abdomen while he was lying flat. There were contusions in the left lumber region with bulging swelling in the left iliac fossa, increased with crying, (Figure 1). Abdomen was soft, but there was tenderness in the left lumber and left iliac fossa regions. An impulse on coughing was present in the left lower quadrant and a defect could be felt in the anterior abdominal wall lateral to the rectus muscle and below the umbilicus.

He was diagnosed as acute handlebar hernia. Routine investigations were within normal limits. Ultrasonography revealed only a defect of $8 \times 4 \mathrm{~cm}$ in the abdominal wall muscles in the left lower quadrant with bowel herniating through the defect. We planned for an open surgery.

An oblique incision was made over the defect. On splitting the external oblique aponeurosis, the rent was visible. The peritoneum was so adherent to the undersurface of external oblique aponeurosis that it got opened with external oblique aponeurosis. No viscus was adherent to the overlying external aponeurosis. Both the external and entire internal oblique aponeurosis was seen passing anterior to the semilunar edge of rectus abdominis very clearly. A plane was created between the internal oblique and the external oblique by sharp dissection with ease.
No attempt was made to separate the transverses abdominis from the internal oblique and mobilise the peritoneum. The gap was repaired with prolene 3-0 without any tension.

Interrupted skin stitches were given with 2-0 silk (Figure 2). The patient had uneventful recovery and has been under regular follow-up for the last 8 months.

\section{Discussion}

Acute abdominal wall hernias caused by traumatic force are exceedingly rare [1]. There are fewer than 50 cases reported in the

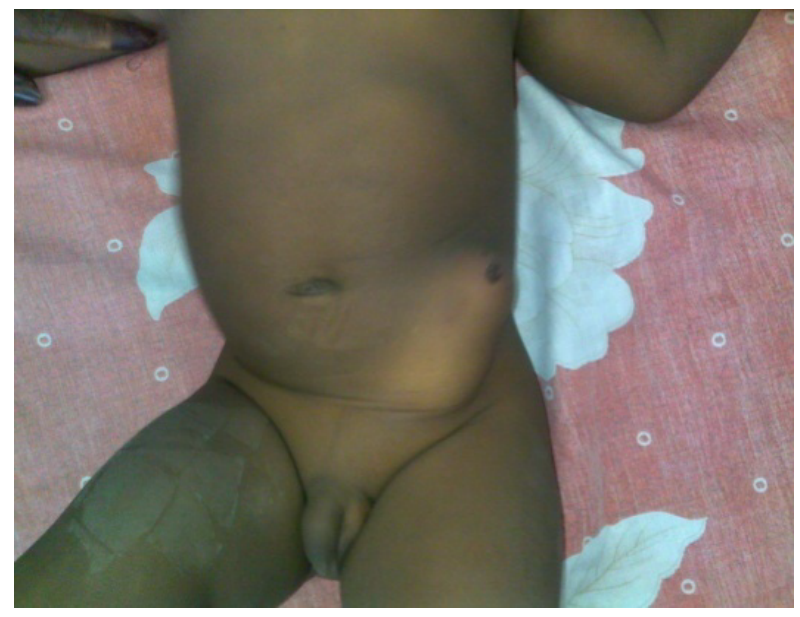

Figure 1. Three years child with acute handlebar abdominal hernia.

Correspondence to: Awad Ali Mohamed Ahmed Alawad, Department of Surgery, Faculty of Medicine, University of Medical Sciences and Technology, Sudan, Tel: 00249912802545; E-mail: awadali82@hotmail.com

Key words: handlebar, hernia

Received: September 03, 2015; Accepted: October 01, 2015; Published: October 05,2015 


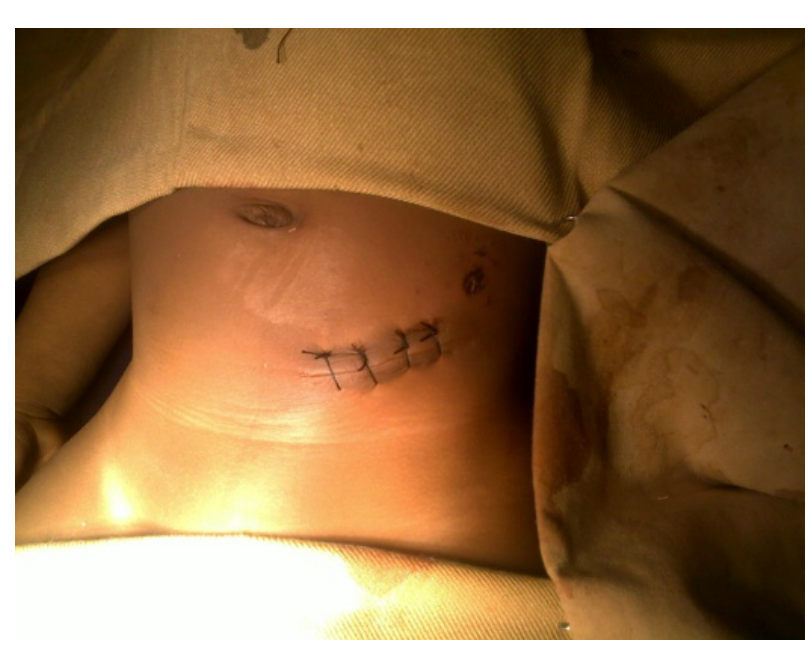

Figure 2. After primary surgical repair.

literature. Handlebar hernias, which are localized defects, are even more infrequent, only 28 cases reported since 1939 [1]. It involves disruption of the abdominal wall muscles, with bowel loop herniated through the defect in the abdominal wall.

Traumatic Abdominal Wall Hernia (TAWH) is postulated to be caused by a force that is sufficient to disrupt the underlying muscles and fascia of the anterior abdominal wall but not the tough and elastic overlying skin. A forceful blow to the abdomen, most commonly due to a handle bar injury or fall from a height has been described as the most common cause of acute TAWH [2]. The abdominal wall defect in handlebar injury is often found in the lower abdomen as in this report, with only two cases reported as occurring in the upper abdomen [3]. It may have major or even lethal complications [2].

The clinical features of TAWH include a history of blunt trauma to the abdomen, abdominal pain and a bulge of the local soft tissue at the ecchymotic area overlying the defect. Physical examination would reveal the swelling with or without tenderness and a palpable defect depending on the phase of presentation to the hospital. An ultrasonography and computed tomography may show subcutaneous bowel loops and the defect. They may also help in differentiating a hernia from a haematoma, define the anatomy of the surrounding abdominal wall and identify other associated injuries. The need for other investigative modalities will depends on other injuries sustained by the patient [4].
Some classification has been proposed for TAWH. Wood et al identified three categories based on the size of the rupture and the cause of the injury. First, the lower quadrant abdominal defects and hernia caused by blunt trauma are most commonly from a handlebar. The second category consists of larger defect hernias that follow motor vehicular crashes. The third category includes intraabdominal bowel herniation into rents in the retroperitoneum [5]. Lane et al. [5] dividedTAWH into two types-low energy injuries following impact on small blunt objects and high energy injuries usually from automobile and pedestrian collisions. Wood et al. [4] classified them depending on the size of the rupture and the cause of the injury: small defects caused by blunt trauma, larger defects sustained during motor vehicle crashes and rarely, intra-abdominal bowel herniation in deceleration injuries (rare) [6].

In this reported case, the presentation was similar to the most of the presentations found in the literature. Ultrasonography was satisfactory for confirming the diagnosis and excluding other associated injuries. Since TAWH occurs in the setting of trauma, a full trauma protocol should be followed. High-energy injuries that are associated with other abdominal injuries require a midline laparotomy to attend to all the injuries. For low-energy injuries the defect can be repaired through an incision placed over the defect since they are not often associated with other abdominal injuries. This case was treated in a similar way without postoperative complications.

\section{Conclusion}

This is a rare type of abdominal hernia that most likely to be seen in the accident and emergency centers. Its diagnosis is simple and so its treatment, unless it is associated with other injuries.

\section{References}

1. Chen HY, Sheu MH, Tseng LM (2005) Bicycle-handlebar hernia: a rare traumatic abdominal wall hernia. J Chin Med Assoc 68: 283-285. [Crossref]

2. Damschen DD, Landercasper J, Cogbill TH, Stolee RT (1994) Acute traumatic abdominal hernia: case reports. J Trauma 36: 273-276. [Crossref]

3. Ogundiran TO, Obamuyide HA, Adesina MA, Ademola AF (2012) Case report of traumatic abdominal wall hernia following blunt motorcycle handlebar injury and review of the literature. Niger J Clin Pract 15: 238-240. [Crossref]

4. Wood RJ, Ney AL, Bubrick MP (1988) Traumatic abdominal hernia: a case report and review of the literature. Am Surg 54: 648-651. [Crossref]

5. Lane CT, Cohen AJ, Cinat ME (2003) Management of traumatic abdominal wall hernia. Am Surg 69: 73-76. [Crossref]

6. Jones BV, Sanchez JA, Vinh D (1989) Acute traumatic abdominal wall hernia. Am J Emerg Med 7: 667-668.

Copyright: (C)2015 Baderalmaarif M. This is an open-access article distributed under the terms of the Creative Commons Attribution License, which permits unrestricted use, distribution, and reproduction in any medium, provided the original author and source are credited. 